\title{
Molecular Methods in Epidemiology of Methicillin Resistant Staphylococcus aureus (MRSA): Advantages, Disadvantages of Different Techniques
}

\section{Judit Szabó*}

Department of Medical Microbiology, University of Debrecen, Hungary

"Corresponding author: Judit Szabó, Department of Medical Microbiology, University of Debrecen, P.O.B. 17, H-4032 Debrecen, Hungary, Tel: +36-52-255-560; Fax: +36-52-255-424; E-mail: szabjud@dote.hu

Rec Date: April 28, 2014; Acc date: July 20, 2014; Pub date: July 22, 2014

Copyright: (C) 2014 Szabó J. This is an open-access article distributed under the terms of the Creative Commons Attribution License, which permits unrestricted use, distribution, and reproduction in any medium, provided the original author and source are credited.

\begin{abstract}
The aim of this review article is to compare molecular typing methods of methicillin resistant Staphylococcus aureus (MRSA) strains. Resistance to methicillin and other beta-lactam antibiotics is caused by the mecA gene, which is situated on a mobile genetic element, the Staphylococcal Cassette Chromosome mec (SCCmec). While hospital acquired (HA)-MRSA strains are typically multi-resistant, community associated (CA)-MRSA strains are by large more susceptible to many antibiotics. More resently multi-resistant livestock associated (LA)-MRSA strains have been recovered from bovine mastitis, but these strains are only prevalent in certain high-risk groups of workers in direct contact with live animals. The epidemiology of MRSA might be investigated by pulsed-field gel electrophoresis (PFGE), multilocus sequence typing (MLST), spa typing, DNA microarray hybridisation and SCCmec typing.
\end{abstract}

Until recently the most useful technique was the PFGE, which has high discriminatory index, but technically difficult and slow, the portability is limited, and multiple nomenclature and misclassification of some lineages also can occur. The mec (SCCmec) typing has standard nomenclature wich able to distinguish between the HA-MRSA and CA-MRSA strains. The spa typing might be a useful tool for epidemiological studies of MRSA, due to its rapidity, accessibility, high throughput, and standardized nomenclature. The MLST has high discriminatory power, defines core genetic population and also has portability and standard nomenclature, but the weaknessess of this method are the low throughput and high cost. Though, the DNA miroarray hybridization test is the most modern technique, which able to detect of presence of a huge number of genomic loci, but due to the high cost and complexity not preferred in routine practice.

Considering all data of these molecular techniques, the most recommended methods are the spa and staphylococcal cassette chromosome mec ( $\mathrm{SCCmec}$ ) typing. Both are informative enough to define particular strain characteristics and utilise standardised nomenclatures, making them applicable globally.

Continuous efforts to follow up the changing epidemiology of MRSA infection in humans and animals are necessary, not only for effective infection control and appropriate antibiotic treatment, but also monitoring the evolution of the MRSA clones.

Keywords: MRSA; Epidemiology; Molecular typing methods

\section{Introduction}

Methicillin-resistant Staphylococcus aureus (MRSA) is a major pathogen worldwide in the hospital and community settings. Hospitalacquired MRSA (HA-MRSA) strains may occur in mainly elderly patients with predisposing factors, such as hospitalization, using of indwelling catheters or surgical procedures, whereas communityacquired (CA-MRSA) strains usually affect healthy and younger people without such risk factors [1]. Furthermore, HA-MRSA and CA-MRSA belong to different genetic lineages. CA-MRSA strains are usually sensitive to antibiotics other than beta-lactams and contain staphylococcal cassette chromosome SCCmec type IV, V or VII, while HA-MRSA are generally multidrug-resistant and harbour larger SCCmec type I, II or III [2]. The presence of type I arginine catabolic mobile element (ACME) has been proposed to contribute to the fitness and transmissibility of the CA-MRSA isolates [3]. More recently, the emergence of an MRSA clone colonizing different animals such as pigs and, more rarely, other farm animals (cattle and poultry) have been reported in Europe. These strains, designated livestock-associated MRSA (LA-MRSA) were mostly found in countries with high density of pig farming such as the Netherlands, Denmark and Germany. Human infections caused by the LA-MRSA strain ST398 have been reported in patients that have had contact with pigs [4].

The most useful molecular methods to categorize isolates into clones and to compare the relevant genetic features of each clone as follows: pulsed-field gel electrophoresis (PFGE), multi-locus sequence typing (MLST), spa typing, staphylococcal cassette chromosome (SCC) mec typing, and DNA microarray hybridisation.

\section{Pulsed-Field Gel Electrophoresis (PFGE)}

The most widely used molecular typing method of MRSA strains is Pulsed-Field Gel Electrophoresis (PFGE). This technique is based on the digestion of bacterial DNA by restriction enzymes. The large 
fragments are running in agarose gel according to their size. The orientation of electric field is changed periodically ("pulsed"). PFGE analysis provides a restriction pattern of bacterial DNA composed of well-defined fragments. This method has proved very successful for the investigation of nosocomial outbreaks and has also been used to identify MRSA clones that have a particular ability to cause major outbreaks and to spread nationally and internationally (epidemic MRSA clones; EMRSA). Though, PFGE has excellent discriminatory power, major disadvantages are that this technique is labor-intensive and difficult to standardize and compare the results from different laboratories [5].

\section{Multilocus Sequence Typing (MLST)}

Multilocus Sequence Typing (MLST) is a highly discriminatory method of characterizing bacterial isolates on the basis of the sequences of approximately 450-bp internal fragments of seven housekeeping gene amplified by PCR. Sequence Type (ST) is obtained based on the alleles identified at each of the seven loci using the SA MLST database. As there are many alleles at each of seven loci, isolates are highly unlikely to have identical allelic profiles by chance, and isolates with the same allelic profile can be assigned as members of the same clone. The major advantage of MLST is the ability to compare the results obtained in different studies. In addition, the data obtained by MLST can be used to address basic questions about the evolutionary and population biology of bacterial species [6]. Disadvantage of MLST is that it uses only seven loci, which limits the ability to detect some switches. It is requires performing PCR and sequencing of the PCR products using an automated sequencer, which is not available in most clinical laboratories. All in all, MLST is not suitable for routine infection control or outbreak investigation due to high cost, labor intensity, and lack of broad access to high-throughput DNA sequencing [6].

\section{Spa Typing}

The method is based on the detection of polymorphic $\mathrm{X}$ region of the protein A gene (spa). By application of the Based Upon Repeat Pattern (BURP) algorithm implemented by the software, spa types with more than five repeats are clustered into different groups, with the calculated cost between members of a group being less than or equal to 6 [7]. It is clear; that spa typing has a high degree of type ability, as well as excellent reproducibility and this method can give interchangeable information. However, to overcome the limitations of spa typing the use of additional markers is indispensable. Additional targets can be SCCmec, lineage-specific virulence or resistance genes, or alternative polymorphic regions of the $S$. aureus chromosome. In most cases a variety of spa types corresponded to a single MLST. Considering that the majority of these genes reside on mobile genetic elements, frequent exchange between different lineages can occur. Another disadvantage is that spa typing is less discriminatory than PFGE [8].

\section{SCCmec Typing}

MRSA strains are characterized by the presence of a large heterologous mobile genetic element called the staphylococcal cassette chromosome mec (SCCmec), which includes the mecA gene, the central element of methicillin resistance. Besides the mec gene complex SCCmec contains the ccr gene complex, which encodes recombinases responsible for the mobility of SCCmec. The remaining parts of the gene complex are called J regions (regions J1, J2, and J3), which constitute nonessential components of the cassette. Although, in some cases these regions harbour additional antibiotic resistance determinants. Multiplex PCR strategy enables the rapid presumptive assignment of all known SCCmec types to MRSA strains, especially CA-MRSA isolates, which are mostly associated with specific SCCmec type [9]. One of the disadvantages of this technique is the complexity of the typing system since SCCmec region is variable and newer types are permanently being defined.

\section{DNA Microarray}

DNA microarray hybridization test is contains covalently immobilized probes specific for approximately 180 genes and 300 alleles of $S$. aureus, including resistance genes, toxins and microbial surface components. The great potentiality of the method is the simultaneous detection of presence of a large number of genomic loci. The test is able to detect potentially new epidemiological markers such as mer operon, which recovered only in the ACME-negative USA300 strains. It can be an alternative molecular typing technique, providing additional features that are complementary to the characterization of the MRSA strains. This new method can evaluate MRSA lineages, but its complexity and cost make it not suitable for clinical purposes at this time [10].

\section{Summary}

Monitoring the global epidemiology of MRSA strains and to standardize the typing techniques is a need for a consensus and to agree on a nomenclature that would allow the evaluate and compare the data at national and international levels. Until now, the most commonly used genotypic technique was the PFGE, recently the spa typing or SCCmec methods are the most preferred.

\section{References}

1. Chavez TT, Decker CF (2008) Health care-associated MRSA versus community-associated MRSA. Dis Mon 54: 763-768.

2. DeLeo FR, Otto M, Kreiswirth BN, Chambers HF (2010) Communityassociated meticillin-resistant Staphylococcus aureus. Lancet 375: 1557-1568.

3. Diep BA, Stone GG, Basuino L (2008) The arginine catabolic mobile element and staphylococcal chromosomal casette mec linkage: convergence of virulence and resistance in the USA300 clone of methicillin-resistant Staphylococcus aureus. J Infect Dis 197:1523-1530.

4. Otter JA, French GL (2010) Molecular epidemiology of communityassociated meticillin-resistant Staphylococcus aureus in Europe. Lancet Infect Dis 10: 227-239.

5. Murchan S, Kaufmann ME, Deplano A (2003) Harmonization of pulsedfield gel electrophoresis protocols for epidemiological typing of strains of methicillin resistant Staphylococcus aureus a single approach developed by consensus in 10 European laboratories and its application for tracing the spread of related strains. J Clin Microbiol 41:1574-1585.

6. Enright MC, Day NP, Davies CE, Peacock SJ, Spratt BG (2000) Multilocus sequence typing for characterization of methicillin-resistant and methicillin-susceptible clones of Staphylococcus aureus. J Clin Microbiol 38: 1008-1015.

7. Strommenger B, Braulke C, Heuck D, Schmidt C, Pasemann B, et al. (2008) spa Typing of Staphylococcus aureus as a frontline tool in epidemiological typing. J Clin Microbiol 46: 574-581.

8. Wisniewska K, Szewczyk A, Piechowicz L (2012) The use of spa and phage typing for characterization of clinical isolates of methicillin- 
Citation: Szabó J (2014) Molecular Methods in Epidemiology of Methicillin Resistant Staphylococcus aureus (MRSA): Advantages, Disadvantages of Different Techniques. J Med Microb Diagn 3: 147. doi:10.4172/2161-0703.1000147

Page 3 of 3

resistant Staphylococcus aureus in the University Clinical enter in Gdansk, Poland. Folia Microbiol 57: 243-249.

9. Milheiriço C, Oliveira DC, de Lencastre H (2007) Update to the multiplex PCR strategy for assignment of mec element types in Staphylococcus aureus. Antimicrob Agents Chemother 51: 3374-3377.
10. Sanchini A, Campanile F, Monaco M, Cafiso V, Rasigade JP, et al. (2011) DNA microarray-based characterisation of Panton-Valentine leukocidinpositive community-acquired methicillin-resistant Staphylococcus aureus from Italy. Eur J Clin Microbiol Infect Dis 30: 1399-1408. 